Hardy-Ramanujan Journal

Vol. 30 (2007) 31-55

\title{
Contributions to the theory of the Hurwitz zeta-function
}

\author{
S. Kanemitsu, Y. Tanigawa, H. Tsukada and M. Yoshimoto
}

Dedicated to Professor Matti Jutila

\begin{abstract}
In this paper we shall give various contributions to the theory of the Hurwitz zeta-function. In $\S 1$ we shall continue our previous study and give integral representations (for the derivatives as well) which give another basis of the theory of gamma and related functions. In $\S 2$ we shall give the sixth proof of the Ramanujan formula with two examples which supersede those results presented in the book of Srivastava and Choi. In $\S 3$ we shall give two more proofs of the closed formula for the integral of the psifunction, thus recovering the recent result of Episona and Moll. Finally, in $\S 4$ we shall give another proof of the functional equation. Hereby we put all existing literature in the hierarchical and historical perspective.
\end{abstract}

Key words: Hurwitz zeta-function, Lerch zeta-function, the Ramanujan formula, integral representation, integral of the psi-function, functional equation

2000 Mathematics Subject Classification: Primary-11M35 ; Secondary-33B15

\section{Introduction}

This is partially a sequel to our previous papers [16]-[18], supplementing the material therein, but in other respects we shall go further and show that many results which are published after [16]-[18] are already contained in our framework.

In $\S 1$ we shall present the integral representation for the partial sum

$$
L_{u}(x, a)=\sum_{0 \leq n \leq x}(n+a)^{u}
$$

of the Hurwitz zeta-function

$$
\zeta(-u, a)=\sum_{n=0}^{\infty}(n+a)^{u} \quad(\operatorname{Re} u<-1)
$$

and for $\zeta$ itself. The proof as presented in [17] is quite simple, but the result is far-reaching and we may even base the whole theory of the gamma and related functions on our results (Theorem 1 and its corollaries). We shall develop this aspect of our theory further [24],[49], and we shall present only a few special cases. The special feature of Theorem 1 is that the derivatives may be computed 
by differentiating with respect to $u$ and the whole results may be inherited (for more details, cf. the introductory remark at the beginning of $\S 1$ ).

In $\S 2$, we are going to give the sixth proof of a far-reaching formula of Ramanujan. This proof, incorporating the structure of the Hurwitz zeta-function as the principal solution of the difference equation, seems one of the most natural ones.

We give two examples which list up summation formulas going beyond those in [45] (cf. Remark 2.1).

In $\S 3$, we shall give two more proofs of the closed formula for the integral $\int_{0}^{z} t^{\lambda} \psi(t+a) \mathrm{d} t$, thus recovering the seemingly most important formula of Episona and Moll [9]. We also give two enlightening remarks, the latter of which clarify the relation between Episona and Moll's results and Mikolás' results.

In $\S 4$, we shall sum up the existing proofs of the functional equation (3.13) for the Hurwitz (Lerch) zeta-function and reveal the hierarchal relationship among them, referring to Laurinčikas and Garunkštis [30] for the Lerch zeta-function aspects. We shall add one more proof of (3.13) based on the Dirac delta-function. Since from the delta-function, we may deduce the Poisson summation formula, we might regard our proof more fundamental.

Thus, "contributions" in the title signifies that we put the existing literature on the Hurwitz zeta-function in their hierarchical and historical perspective, with our recent contributions [16], [17], [18] as touchstones.

We shall use the following notation and known results freely in what follows, without referring to them on each occasion (all can be found e.g. in Erdélyi [11]).

\section{Notation:}

$$
\zeta(s, a)=\sum_{n=0}^{\infty} \frac{1}{(n+a)^{s}}, \quad \operatorname{Re} s=\sigma>1, \quad a>0
$$

- the Hurwitz zeta-function;

$$
\zeta(s)=\zeta(s, 1)=\sum_{n=1}^{\infty} \frac{1}{n^{s}}, \quad \sigma>1
$$

- the Riemann zeta-function (both are meromorphically continued over the whole plane with a simple pole at $s=1)$;

$$
l_{s}(a)=\sum_{n=1}^{\infty} \frac{e^{2 \pi i n a}}{n^{s}}, \quad \sigma>1, \quad 0<a(<1)
$$

- the Lerch zeta-function or the polylogarithm function;

$$
\Gamma(s)=\int_{0}^{\infty} e^{-t} t^{s-1} \mathrm{~d} t, \quad \sigma>0
$$

- the gamma function;

$$
\gamma(s, a)=\int_{0}^{a} e^{-t} t^{s-1} \mathrm{~d} t, \quad \Gamma(s, a)=\int_{a}^{\infty} e^{-t} t^{s-1} \mathrm{~d} t
$$


- the incomplete gamma functions of the 1st and the 2nd kind, which satisfy $\gamma(s, a)+\Gamma(s, a)=\Gamma(s)$

$$
\psi(s)=\frac{\Gamma^{\prime}(s)}{\Gamma(s)}=\frac{\mathrm{d}}{\mathrm{d} s} \log \Gamma(s)
$$

- the psi function or the Euler digamma function;

$$
H_{n}=\psi(n+1)-\psi(1)=\psi(n+1)+\gamma=\sum_{k=1}^{n} \frac{1}{k}
$$

- the $n$-th harmonic number, where $\gamma$ signifies Euler's constant (see below as the Laurent constant $\left.\gamma_{0}(1)=-\psi(1)=-\gamma\right)$.

$$
B_{n}(z)=\sum_{k=0}^{n}\left(\begin{array}{l}
n \\
k
\end{array}\right) B_{k} z^{n-k}
$$

- the $n$-th Bernoulli polynomial with $B_{k}$ the $k$-th Bernoulli number defined through

$$
\begin{gathered}
\frac{z}{e^{z}-1}=\sum_{k=0}^{\infty} \frac{B_{k}}{k !} z^{k} \quad|z|<2 \pi ; \\
\bar{B}_{n}(z)=B_{n}(\{z\})=B_{n}(z-[z]) \quad \text { for } z \in \mathbb{R}
\end{gathered}
$$

- the $n$-th periodic Bernoulli polynomial, with $[x]$ and $\{x\}$ signifying the integral and fractional parts of $x$, respectively.

\section{Known formulas:}

$$
\begin{gathered}
B_{m}(z)=-m \zeta(1-m, z), \quad m \in \mathbb{N}, \\
\psi^{(m)}(z)=(-1)^{m+1} m ! \zeta(m+1, z), \quad m \in \mathbb{N} .
\end{gathered}
$$

The Laurent expansion of $\zeta(s, a)$ at $s=1$ is given by

$$
\zeta(s, a)=\frac{1}{s-1}-\psi(a)+\sum_{n=1}^{\infty} \frac{(-1)^{n} \gamma_{n}(a)}{n !}(s-1)^{n}, \quad s \rightarrow 1 .
$$

The finite difference equation satisfied by the Hurwitz zeta-function:

$$
\zeta(s, a+1)-\zeta(s, a)=-a^{-s} .
$$

The addition formula for the Bernoulli polynomial

$$
B_{n}(x+y)=\sum_{k=0}^{n}\left(\begin{array}{l}
n \\
k
\end{array}\right) B_{k}(x) y^{n-k} .
$$




\section{Integral representations}

For complex $u$ and $a$ and $x \geq 0$ let

$$
L_{u}(x, a)=\sum_{0 \leq n \leq x}(n+a)^{u},
$$

where for negative values of $u$, the possible value of $n$ for which $n+a=0$ is to be excluded.

We proved in [17] an integral representation for $L_{u}(x, a)$ (restated as Theorem 1 below), which has the following far-reaching features shared by the derivatives $\frac{\partial^{k}}{\partial u^{k}} L_{u}(x, a)$ as well, i.e. all statements about the function in $u\left(L_{u}(x, a)\right.$ and $\zeta(-u, a))$ are valid for their derivatives as in the form of (i) below.

(i) It gives an analytic expression for $L_{u}(x, a)$, which entails an integral representation for each derivative $\frac{\partial^{k}}{\partial u^{k}} L_{u}(x, a)=\sum_{0 \leq n \leq x}(n+a)^{u} \log ^{k}(n+a)$ (the differentiation of the integral being carried out under the integral sign).

(ii) It gives an asymptotic formula for $L_{u}(x, a)$ in $x$ by estimating the integral trivially, which is feasible for applications in the divisor problems.

(iii) It gives a generic definition of $\zeta(-u, a)$ for $u \neq-1$ (and for $\gamma_{0}(a):=$ $-\psi(a)$ for $u=-1)$.

(iv) It gives an integral representation for the associated Hurwitz zetafunction $\zeta(-u, a)$ (and its derivatives $\frac{\partial^{k}}{\partial u^{k}} \zeta(-u, a)=\zeta^{(k)}(-u, a)$ ) for $u \neq-1$, and for $u=-1$, it gives an analytic expression for the generalized Euler constant $\gamma_{k}(a)$ (the $k$-th Laurent coefficient of $\zeta(s, a)$ at $s=1$ ), which follows by simply putting $x=0$ in the integral representation.

(v) The integral representation for $\zeta(s, a)$ (or $\left.\gamma_{k}(a)\right)$ in (iii) yields an asymptotic formula for the $\zeta(s, a+z)$ in $z$ with Bernoulli polynomial coefficients (Theorem 2 [17]) as given by M. Katsurada [26].

Theorem 1 (Integral Representations). Let

$$
L_{u}(x, a)=\sum_{0 \leq n \leq x}(n+a)^{u},
$$

where $u$ is a complex variable, and $a$ is another complex variable, and where in the case $\operatorname{Re} u<0$, the possible value of $n$ which is equal to $-a$ is to be excluded. Then, for any $l \in \mathbb{N}$ with $l>\operatorname{Re} u+1$ and for any $x \geq 0$,

$$
\begin{aligned}
L_{u}(x, a)= & \sum_{r=1}^{l} \frac{\Gamma(u+1)}{\Gamma(u+2-r)} \frac{(-1)^{r}}{r !} \bar{B}_{r}(x)(x+a)^{u-r+1} \\
& +\frac{(-1)^{l}}{l !} \frac{\Gamma(u+1)}{\Gamma(u+1-l)} \int_{x}^{\infty} \bar{B}_{l}(t)(t+a)^{u-l} \mathrm{~d} t \\
& + \begin{cases}\frac{1}{u+1}(x+a)^{u+1}+\zeta(-u, a), & u \neq-1 \\
\log (x+a)-\psi(a), & u=-1 .\end{cases}
\end{aligned}
$$


Also the asymptotic formula

$$
\begin{aligned}
L_{u}(x, a)= & \sum_{r=1}^{l} \frac{(-1)^{r}}{r}\left(\begin{array}{c}
u \\
r-1
\end{array}\right) \bar{B}_{r}(x)(x+a)^{u-r+1}+O\left(x^{\operatorname{Re}(u)-l}\right) \\
& + \begin{cases}\frac{1}{u+1}(x+a)^{u+1}+\zeta(-u, a), & u \neq-1 \\
\log (x+a)-\psi(a), & u=-1\end{cases}
\end{aligned}
$$

holds true as $x \rightarrow \infty$.

Furthermore, the integral representation

$$
\begin{aligned}
\zeta(-u, a)= & a^{u}-\frac{1}{u+1} a^{u+1} \\
& -\sum_{r=1}^{l} \frac{(-1)^{r}}{r}\left(\begin{array}{c}
u \\
r-1
\end{array}\right) B_{r} a^{u-r+1} \\
& +(-1)^{l+1}\left(\begin{array}{c}
u \\
l
\end{array}\right) \int_{0}^{\infty} \bar{B}_{l}(t)(t+a)^{u-l} \mathrm{~d} t,
\end{aligned}
$$

which follows from (1.1) by putting $x=0$, holds true for all complex $u \neq-1$, where $l$ can be any natural number subject only to the condition that $l>\operatorname{Re} u+1$.

Since the integrals appearing in Theorem 1 are analytic in the region, $\operatorname{Re} u<$ $1-l$, we may differentiate (1.1) and (1.3) in $u$ there. We state the counterparts of (1.1) as the following corollaries:

Corollary 1. For any complex $u$ and $a>0$,

$$
\begin{aligned}
& \frac{\mathrm{d}}{\mathrm{d} u} L_{u}(x, a) \\
& =\sum_{0 \leq n \leq x}(n+a)^{u} \log (n+a) \\
& =\sum_{r=1}^{l} \frac{(-1)^{r}}{r !} \bar{B}_{r}(x)(x+a)^{u-r+1} \\
& \quad \times \frac{\Gamma(u+1)}{\Gamma(u+2-r)}\{\psi(u+1)-\psi(u+2-r)+\log (x+a)\} \\
& +\frac{(-1)^{l}}{l !} \int_{x}^{\infty} \bar{B}_{l}(t)(t+a)^{u-l} \\
& \quad \times \frac{\Gamma(u+1)}{\Gamma(u+1-l)}\{\psi(u+1)-\psi(u+1-l)+\log (t+a)\} \mathrm{d} t
\end{aligned}
$$


Corollary 2. For any complex $u$ and $a>0$,

$$
\begin{aligned}
& \frac{\mathrm{d}^{2}}{\mathrm{~d} u^{2}} L_{u}(x, a) \\
& =\sum_{r=1}^{l} \frac{(-1)^{r}}{r !} \bar{B}_{r}(x)(x+a)^{u-r+1} \\
& \quad \times \frac{\Gamma(u+1)}{\Gamma(u+2-r)}\left[\{\psi(u+1)-\psi(u+2-r)+\log (x+a)\}^{2}\right. \\
& \left.\quad+\psi^{\prime}(u+1)-\psi^{\prime}(u+2-r)\right] \\
& +\frac{(-1)^{l}}{l !} \int_{x}^{\infty} \bar{B}_{l}(t)(t+a)^{u-l} \\
& \quad \times \frac{\Gamma(u+1)}{\Gamma(u+1-l)}\left[\{\psi(u+1)-\psi(u+1-l)+\log (t+a)\}^{2}\right.
\end{aligned}
$$

We note that Theorem 1 is the most informative for $L_{u}(x, a)$, so are Corollaries 1 and 2 for $\frac{\partial}{\partial u} L_{u}(x, a)$ and $\frac{\partial^{2}}{\partial u^{2}} L_{u}(x, a)$, respectively. Care should be taken in interpreting the coefficients like $\frac{\Gamma(u+1)}{\Gamma(u+1-l)}(\psi(u+1)-\psi(u+1-l))$ when $u$ is a negative integer; it is to be taken as one without singularities (cf. (1.7) below).

We shall illustrate these by the first derivative $\left(\frac{\partial}{\partial u} L_{u}(x, a)\right.$ or $\left.-\zeta^{\prime}(-u, a)\right)$ in the special case of $u=m, m \in \mathbb{N} \cup\{0\}$. For $\mathbb{N} \ni l>m+1$, Corollary 1 eventually yields (cf. [33])

$$
\begin{aligned}
-\zeta^{\prime}(-m, a)= & \lim _{N \rightarrow \infty}\left(\sum_{n=0}^{N}(n+a)^{m} \log (n+a)\right. \\
& -\frac{1}{m+1}(N+a)^{m+1} \log (N+a)+\frac{1}{(m+1)^{2}}(N+a)^{m+1} \\
& -\frac{1}{2}(N+a)^{m} \log (N+a)-\sum_{r=2}^{m+1}\left(\begin{array}{c}
m \\
r-1
\end{array}\right) \frac{B_{r}}{r !} \\
& \left.\cdot\left(\frac{1}{m}+\cdots+\frac{1}{m-r+2}+\log (N+a)\right)(N+a)^{m-r+1}\right) .
\end{aligned}
$$


and

$$
\begin{aligned}
& \zeta^{\prime}(-m, a) \\
&= \frac{1}{m+1} a^{m+1} \log a \\
&-\frac{1}{(m+1)^{2}} a^{m+1}-\frac{1}{2} a^{m} \log a+\frac{1}{12} a^{m-1} \log a \\
&+\sum_{r=4}^{m+1} \frac{B_{r}}{r}\left(\sum_{j=0}^{r-2}(-1)^{j}\left(\begin{array}{c}
m \\
j
\end{array}\right) \frac{1}{r-1-j}+\left(\begin{array}{c}
m \\
r-1
\end{array}\right) \log a\right) \\
&+\frac{1}{m+1} \sum_{r=m+2}^{l} B_{r}\left(\sum_{j=0}^{r-1}(-1)^{j}\left(\begin{array}{c}
r-m-2 \\
j
\end{array}\right) \frac{1}{r-j}\right) a^{m-r+1} \\
&+(-1)^{l+1} \int_{0}^{\infty}\left(\sum_{j=0}^{l-1}(-1)^{j}\left(\begin{array}{c}
l-m-1 \\
j
\end{array}\right) \frac{1}{l-j} \bar{B}_{l}(t)(t+a)^{m-l}\right) \mathrm{d} t
\end{aligned}
$$

where (1.6) and (1.7) correspond to (iii) and (iv), respectively.

We shall show that, in view of Lerch's relation

$$
\log \frac{\Gamma(a)}{\sqrt{2 \pi}}=\zeta^{\prime}(0, a)
$$

(1.6) with $m=0$ gives Euler's product formula (??) for $\Gamma(a)$.

Indeed,we eventually tramform (1.6) into

$$
\begin{gathered}
-\log \frac{\Gamma(a)}{\sqrt{2 \pi}}=\lim _{N \rightarrow \infty}\left(\sum_{n=0}^{N} \log (n+a)-\left(N+\frac{1}{2}\right) \log N\right. \\
+N-a \log N) .
\end{gathered}
$$

Subtracting from (1.8) its special case with $a=1$ yields

$$
-\log \Gamma(a)=\lim _{N \rightarrow \infty}\left(\log a+\sum_{n=1}^{N} \log \frac{n+a}{n}-a \log N\right) .
$$

whence we conclude that

$$
\frac{1}{\Gamma(a)}=a \prod_{n=1}^{\infty}\left\{\left(1+\frac{1}{n}\right)^{-a}\left(1+\frac{a}{n}\right)\right\},
$$

i.e. Euler's product formula (cf. [45, (7), p. 2]).

Remark 1.1. Our procedure is a reverse to that of Berndt [7] in which he starts from one of the equivalent definitions of the gamma function given by 


$$
\log \Gamma(a)=\lim _{N \rightarrow \infty}\left(-\sum_{n=0}^{N} \log (n+a)+\sum_{n=1}^{N} \log n+a \log (N+1)\right)
$$

and deduces Lerch's formula by comparing (1.11) with (1.8). Of course, we can cover (1.11) in the same way as above.

We may recover Deninger's Theorem 2.3 [8], especially, the Gaussian representation

$$
\begin{aligned}
-\zeta^{\prime \prime}(0, a)= & -\zeta^{\prime \prime}(0)-\log ^{2} a \\
& +\lim _{N \rightarrow \infty}\left(a \log ^{2} N-\sum_{n=1}^{N}\left(\log ^{2}(n+a)-\log ^{2} n\right)\right) .
\end{aligned}
$$

by Corollary 2, which eventually leads to

$$
\begin{aligned}
-\zeta^{\prime \prime}(0, a)= & -\zeta^{\prime \prime}(0)-\log ^{2} a+a \log ^{2} N \\
& -\sum_{n=1}^{N}\left(\log ^{2}(n+a)-\log ^{2} n\right)+O\left(\frac{\log ^{2} N}{N}\right)
\end{aligned}
$$

We may also recover the Weierstrass representation $([8,(2.3 .2)])$ by Corollary 1 with $u=-1, a=1$ and $x=N \in \mathbb{N}$ (we write $N$ for $N+1$ ):

$$
\gamma_{1}=\sum_{n=1}^{N} \frac{\log n}{n}-\frac{1}{2} \log ^{2} N+O\left(\frac{\log N}{N}\right) .
$$

(cf. $[17,(8)])$.

Solving (1.14) for $\log ^{2} N$ and substituting it in (1.13), we deduce that

$$
\begin{aligned}
-\zeta^{\prime \prime}(0, a)= & -\zeta^{\prime \prime}(0)-2 \gamma_{1} \log a-\log ^{2} a \\
& -\sum_{n=1}^{N}\left(\log ^{2}(n+a)-\log ^{2} n-2 \frac{\log n}{n}\right)+O\left(\frac{\log ^{2} N}{N}\right),
\end{aligned}
$$

which gives the Weierstrass representation.

\section{A formula of Ramanujan}

In this section we are going to give the sixth proof of the fundamental summation formula (Ramanujan's formula) based on the use of finite differences, which has been applied successfully in recent researches [18], [19]. 
Theorem 2. For $0 \leq \lambda \in \mathbb{Z}$ and $|z|<|\alpha|$ we have

$$
\begin{aligned}
\sum_{m=2}^{\infty} \frac{\zeta(m, \alpha)}{m+\lambda} z^{m+\lambda}= & \sum_{k=0}^{\lambda}\left(\begin{array}{l}
\lambda \\
k
\end{array}\right) \zeta^{\prime}(-k, \alpha-z) z^{\lambda-k} \\
& -\zeta^{\prime}(-\lambda, \alpha)-\sum_{k=1}^{\lambda} \frac{1}{k} \zeta(k-\lambda, \alpha) z^{k} \\
& +\frac{1}{\lambda+1}\left(\psi(\alpha)-H_{\lambda}\right) z^{\lambda+1} .
\end{aligned}
$$

Proof. Let $\Delta_{\alpha} f(\alpha)=f(\alpha+1)-f(\alpha)$ be the difference operator. We apply this to the sum $S$ on the LHS of (2.1) to obtain

$$
\begin{aligned}
\Delta_{\alpha} S=\Delta_{\alpha} \sum_{m=2}^{\infty} \frac{\zeta(m, \alpha)}{m+\lambda} z^{m+\lambda} & =-\sum_{m=2}^{\infty} \frac{\alpha^{-m}}{m+\lambda} z^{m+\lambda} \\
& =-\alpha^{\lambda} \sum_{m=\lambda+2}^{\infty} \frac{1}{m}\left(\frac{z}{\alpha}\right)^{m}
\end{aligned}
$$

The resulting infinite series is nothing but

$$
-\log \left(1-\frac{z}{\alpha}\right)-\sum_{m=1}^{\lambda+1} \frac{1}{m}\left(\frac{z}{\alpha}\right)^{m},
$$

or

$$
-\left(\log (\alpha-z)-\log \alpha+\sum_{m=1}^{\lambda+1} \frac{1}{m}\left(\frac{z}{\alpha}\right)^{m}\right),
$$

whence

$$
\Delta_{\alpha} S=\alpha^{\lambda} \log (\alpha-z)-\alpha^{\lambda} \log \alpha+\sum_{m=1}^{\lambda+1} \frac{\alpha^{\lambda-m}}{m} z^{m} .
$$

Rewriting the first term on the RHS of (2.2) in the form $\sum_{k=0}^{\lambda}\left(\begin{array}{l}\lambda \\ k\end{array}\right) z^{\lambda-k}(\alpha-$ $z)^{k} \log (\alpha-z)$ and telescoping (2.2), thereby noting that

$$
\zeta^{\prime}(s, \alpha+1)-\zeta^{\prime}(s, \alpha)=\alpha^{-s} \log \alpha,
$$

we get

$$
\begin{aligned}
S= & \sum_{k=0}^{\lambda}\left(\begin{array}{l}
\lambda \\
k
\end{array}\right) \zeta^{\prime}(-k, \alpha-z) z^{\lambda-k}-\zeta^{\prime}(-\lambda, \alpha) \\
& -\sum_{k=1}^{\lambda} \frac{1}{k} \zeta(k-\lambda, \alpha) z^{k}+\frac{\psi(\alpha)}{\lambda+1} z^{\lambda+1}+f(z, \alpha),
\end{aligned}
$$


where $f(z, \alpha)$ is the function satisfying the conditions

$$
\Delta_{\alpha} f(z, \alpha)=0
$$

and

$$
f(0, \alpha)=0 .
$$

It remains to determine $f(z, \alpha)$ (to be $\left.-\frac{H_{\lambda}}{\lambda+1} z^{\lambda+1}\right)$. First note that

$$
\begin{aligned}
\frac{\mathrm{d}}{\mathrm{d} z} \zeta^{\prime}(-k, \alpha-z) & =\left.\frac{\partial}{\partial s} \frac{\partial}{\partial z} \zeta(s, \alpha-z)\right|_{s=-k} \\
& =\left.\frac{\partial}{\partial s} s \zeta(s+1, \alpha-z)\right|_{s=-k} \\
& = \begin{cases}\zeta(1-k, \alpha-z)-k \zeta^{\prime}(1-k, \alpha-z), & k \in \mathbb{N} \\
-\psi(\alpha-z), & k=0 .\end{cases}
\end{aligned}
$$

With this in mind, we differentiate (2.3) with respect to $z$ to obtain

$$
\begin{aligned}
\frac{\partial}{\partial z} S= & \sum_{k=0}^{\lambda-1}\left(\begin{array}{l}
\lambda \\
k
\end{array}\right)(\lambda-k) \zeta^{\prime}(-k, \alpha-z) z^{\lambda-k-1} \\
& +\sum_{k=1}^{\lambda}\left(\begin{array}{l}
\lambda \\
k
\end{array}\right) \zeta(1-k, \alpha-z) z^{\lambda-k}-\sum_{k=1}^{\lambda}\left(\begin{array}{l}
\lambda \\
k
\end{array}\right) \zeta^{\prime}(1-k, \alpha-z) z^{\lambda-k} \\
& -\psi(\alpha-z) z^{\lambda}-\sum_{k=1}^{\lambda} \zeta(k-\lambda, \alpha) z^{k-1}+\psi(\alpha) z^{-\lambda}+\frac{\partial}{\partial z} f(z, \alpha) .
\end{aligned}
$$

We note that the two sums on the RHS of (2.6) containing $\zeta^{\prime}$ cancel each other, while the second sum, say $S_{2}$, becomes, in view of the addition formula,

$$
\begin{aligned}
S_{2} & =-\sum_{k=1}^{\lambda}\left(\begin{array}{l}
\lambda \\
k
\end{array}\right) \frac{1}{k} B_{k}(\alpha-z) z^{\lambda-k} \\
& =-\sum_{k=1}^{\lambda}\left(\begin{array}{l}
\lambda \\
k
\end{array}\right) \frac{z^{\lambda-k}}{k} \sum_{l=1}^{k}\left(\begin{array}{l}
k \\
l
\end{array}\right) B_{l}(\alpha)(-z)^{k-l}-\sum_{k=1}^{\lambda}\left(\begin{array}{l}
\lambda \\
k
\end{array}\right) \frac{z^{\lambda-k}}{k}(-z)^{k} \\
& =S_{2,1}+S_{2,2},
\end{aligned}
$$

say. Using

$$
\left(\begin{array}{l}
\lambda \\
k
\end{array}\right)\left(\begin{array}{l}
k \\
l
\end{array}\right)=\left(\begin{array}{l}
\lambda \\
l
\end{array}\right)\left(\begin{array}{l}
\lambda-l \\
k-l
\end{array}\right)
$$

and changing the order of summation in $S_{2,1}$, we have

$$
S_{2,1}=-\sum_{l=1}^{\lambda}\left(\begin{array}{c}
\lambda \\
l
\end{array}\right) B_{l}(\alpha) z^{\lambda-l} \sum_{k=0}^{\lambda-l}\left(\begin{array}{c}
\lambda-l \\
k
\end{array}\right) \frac{(-1)^{k}}{k+l} .
$$


Invoking the formula

$$
\sum_{k=0}^{K}\left(\begin{array}{c}
K \\
k
\end{array}\right) \frac{(-1)^{k}}{k+l}=\frac{K ! \Gamma(l)}{\Gamma(l+K+1)}
$$

we deduce that

$$
S_{2,1}=-\sum_{l=1}^{\lambda} \frac{B_{l}(\alpha)}{l} z^{\lambda-l}=\sum_{l=1}^{\lambda} \zeta(1-l, \alpha) z^{\lambda-l}=\sum_{l=1}^{\lambda} \zeta(l-\lambda, \alpha) z^{l-1} .
$$

For $S_{2,2}$, we use another formula

$$
\sum_{k=1}^{\lambda}\left(\begin{array}{l}
\lambda \\
k
\end{array}\right) \frac{(-1)^{k}}{k}=H_{\lambda}
$$

to obtain

$$
S_{2,2}=H_{\lambda} z^{\lambda} .
$$

From (2.7), (2.8) and (2.9) it follows that

$$
S_{2}=\sum_{l=1}^{\lambda} \zeta(l-\lambda, \alpha) z^{l-1}+H_{\lambda} z^{\lambda}
$$

Substituting (2.10) in (2.6), we conclude that

$$
\frac{\partial}{\partial z} S=-\psi(\alpha-z) z^{\lambda}+\psi(\alpha) z^{\lambda}+H_{\lambda} z^{\lambda}+\frac{\partial}{\partial z} f(z, \alpha) .
$$

On the other hand, from (2.3) we know that

$$
\frac{\partial}{\partial z} S=-(\psi(\alpha-z)-\psi(\alpha)) z^{\lambda} .
$$

Hence, comparing (2.11) and (2.12), we obtain

$$
\frac{\partial}{\partial z} f(z, \alpha)=-H_{\lambda} z^{\lambda}
$$

whence

$$
f(z, \alpha)=-\frac{H_{\lambda}}{\lambda+1} z^{\lambda+1}+C .
$$

By condition (2.5), $C=0$, and

$$
f(z, \alpha)=-\frac{H_{\lambda}}{\lambda+1} z^{\lambda+1} .
$$

Substitution of (2.13) into (2.3) completes the proof. 
Example 1. (i) ([17, Corollary 1], [45, (720), p. 250])

$$
\begin{aligned}
\sum_{m=2}^{\infty} & \frac{(-1)^{m} \zeta(m, \alpha)}{m(m+1)} z^{m+1} \\
= & \log \Gamma_{2}(z+\alpha)+(z+\alpha-1) \log \Gamma(z+\alpha) \\
& -\log \Gamma_{2}(\alpha)-(\alpha-1) \log \Gamma(\alpha) \\
& -\left(\log \Gamma(\alpha)+\alpha-\frac{1}{2} \log 2 \pi-\frac{1}{2}\right) z-\frac{1}{2}(\psi(\alpha)+1) z^{2}
\end{aligned}
$$

(ii) (cf. [45, (569), p. 227 and (712), p. 248], [47, (4), p. 91])

$$
\begin{aligned}
\sum_{m=2}^{\infty} & \frac{(-1)^{m} \zeta(m, \alpha)}{m(m+1)(m+2)} z^{m+2} \\
= & \log \Gamma_{3}(z+\alpha)+\left(z+\alpha-\frac{3}{2}\right) \log \Gamma_{2}(z+\alpha)+\frac{1}{2}(z+\alpha-1)^{2} \log \Gamma(z+\alpha) \\
& -\log \Gamma_{3}(\alpha)-\left(\alpha-\frac{3}{2}\right) \log \Gamma_{2}(\alpha)-\frac{1}{2}(\alpha-1)^{2} \log \Gamma(\alpha) \\
& -\left(\log \Gamma_{2}(\alpha)+(\alpha-1) \log \Gamma(\alpha)+\frac{1}{4} \alpha^{2}-\frac{1}{4} \alpha+\zeta^{\prime}(-1)+\frac{1}{24}\right) z \\
& -\frac{1}{2}\left(\log \Gamma(\alpha)+\frac{3}{2} \alpha-\frac{1}{2} \log 2 \pi-\frac{3}{4}\right) z^{2}-\frac{1}{6}\left(\psi(\alpha)+\frac{3}{2}\right) z^{3},
\end{aligned}
$$

(iii) (cf. [45, (569), p. 227 and (712), p. 248] and [47, (6), p. 92])

$$
\begin{aligned}
\sum_{m=2}^{\infty} & \frac{(-1)^{m} \zeta(m, \alpha)}{m(m+1)(m+2)(m+3)} z^{m+3} \\
= & \log \Gamma_{4}(z+\alpha)+(z+\alpha-2) \log \Gamma_{3}(z+\alpha) \\
& +\frac{1}{6}\left(3(z+\alpha)^{2}-9(z+\alpha)+7\right) \log \Gamma_{2}(z+\alpha) \\
& +\frac{1}{6}(z+\alpha-1)^{3} \log \Gamma(z+\alpha) \\
- & \log \Gamma_{4}(\alpha)-(\alpha-2) \log \Gamma_{3}(\alpha)-\frac{1}{6}\left(3 \alpha^{2}-9 \alpha+7\right) \log \Gamma_{2}(\alpha) \\
- & \frac{1}{6}(\alpha-1)^{3} \log \Gamma(\alpha) \\
- & \frac{1}{2}\left(2 \log \Gamma_{3}(\alpha)+(2 \alpha-3) \log \Gamma_{2}(\alpha)+(\alpha-1)^{2} \log \Gamma(\alpha)\right. \\
& \left.\quad+\frac{1}{9} \alpha^{3}-\frac{1}{6} \alpha^{2}+\frac{1}{18} \alpha+\zeta^{\prime}(-2)\right) z \\
- & \frac{1}{2}\left(\log \Gamma_{2}(\alpha)+(\alpha-1) \log \Gamma(\alpha)+\frac{5}{12} \alpha^{2}-\frac{5}{12} \alpha+\zeta^{\prime}(-1)+\frac{5}{72}\right) z^{2} \\
- & \frac{1}{6}\left(\log \Gamma(\alpha)+\frac{11}{6} \alpha-\frac{1}{2} \log 2 \pi-\frac{11}{12}\right) z^{3}-\frac{1}{24}\left(\psi(\alpha)+\frac{11}{6}\right) z^{4} .
\end{aligned}
$$


Example 2. ([17, Corollary 2]).

(i)

$$
\begin{aligned}
& \sum_{m=2}^{\infty} \frac{\zeta(m, \alpha)}{m+1} z^{m+1} \\
& =(\alpha-1) \log \Gamma(\alpha-z)+\log \Gamma_{2}(\alpha-z)-(\alpha-1) \log \Gamma(\alpha)+\log \Gamma_{2}(\alpha) \\
& \quad-\left(\alpha+\frac{1}{2} \log 2 \pi-\frac{1}{2}\right) z+\frac{1}{2}(\psi(\alpha)-1) z^{2}, \\
& \sum_{m=2}^{\infty} \frac{\zeta(m, \alpha)}{m+2} z^{m+2} \\
& =(\alpha-1)^{2} \log \Gamma(\alpha-z)+(2 \alpha-3) \log \Gamma_{2}(\alpha-z)+2 \log \Gamma_{3}(\alpha-z) \\
& \quad-(\alpha-1)^{2} \log \Gamma(\alpha)+(2 \alpha-3) \log \Gamma_{2}(\alpha)-2 \log \Gamma_{3}(\alpha) \\
& \quad+\frac{1}{2}\left(\alpha^{2}-\alpha+4 \zeta^{\prime}(-1)+\frac{1}{6}\right) z-\frac{1}{2}\left(\alpha+\log 2 \pi-\frac{1}{2}\right) z^{2} \\
& \quad+\frac{1}{3}\left(\psi(\alpha)-\frac{3}{2}\right) z^{3},
\end{aligned}
$$

(ii) $\quad \sum_{m=2}^{\infty} \frac{\zeta(m, \alpha)}{m+2} z^{m+2}$

(iii)

$$
\begin{aligned}
& \sum_{m=2}^{\infty} \frac{\zeta(m, \alpha)}{m+3} z^{m+3} \\
& =(\alpha-1)^{3} \log \Gamma(\alpha-z)+\left(3 \alpha^{2}-9 \alpha+7\right) \log \Gamma_{2}(\alpha-z) \\
& \quad+(6 \alpha-12) \log \Gamma_{3}(\alpha-z)+6 \log \Gamma_{4}(\alpha-z) \\
& \quad-(\alpha-1)^{3} \log \Gamma(\alpha)-\left(3 \alpha^{2}-9 \alpha+7\right) \log \Gamma_{2}(\alpha) \\
& \quad-(6 \alpha-12) \log \Gamma_{3}(\alpha)-6 \log \Gamma_{4}(\alpha) \\
& \quad+\frac{1}{3}\left(\alpha^{3}-\frac{3}{2} \alpha^{2}+\frac{1}{2} \alpha-\frac{9}{4 \pi^{2}} \zeta(3)\right) z \\
& \quad+\frac{1}{4}\left(\alpha^{2}-\alpha+12 \zeta^{\prime}(-1)+\frac{1}{6}\right) z^{2} \\
& \quad-\frac{1}{3}\left(\alpha+\frac{3}{2} \log 2 \pi-\frac{1}{2}\right) z^{3}+\frac{1}{4}\left(\psi(\alpha)-\frac{11}{6}\right) z^{4} .
\end{aligned}
$$

Remark 2.1. (i) As should be clear from our papers [16], [17], [18], the formulas in Examples 1 and 2 are mutual counterparts which transform into the other under a combinatorial principle. Unfortunately, this situation has never been noticed in other works and there have appeared many papers rediscovering these series. Also, as remarked in [20, 21] those formulas in Example 1 are not so rapidly convergent and those coming from Ramanujan are more rapidly convergent.

(ii) This supplements Remark 3 of [17], where comparison was made of the works of Wilton [47] and Srivastava and Choi [45].

Regarding Example 1, (ii), while Srivastava and Choi's formula ([45, (569), p. 227]) might not be viewed as a closed form, involving the unknown integral of Barnes' $G$-function, ([45, (712), p. 248]) equals Wilton's [47, (4), p. 92]. 
Regarding Example 1, (iii), which is the highest summit of [45], superseding previous minor ones ([45, (713), (714), (715)]) corresponds to Wilton's [47, (6), p. 92].

\section{Some definite integrals}

On p. 455, lines 7-11 of [10], Episona and Moll state "The derivative of the Hurwitz zeta function has appeared before in connection with integrals of $\ln \Gamma(q)$ [6], and in a number of related contexts, such as the studies of polygamma functions of negative order [1], the Barnes function [2] and the multiple gamma function [10], and other unrelated (underlined by us) ones such as the evaluation of sums of the type $\sum_{m \geq 2} z^{m} \zeta(m, \alpha)$ [7]". Here "[7]" refers to our paper [16].

The last part of this statement is not very precise in the sense that although in the evaluation of the sums of the type mentioned, the derivative of the Hurwitz zeta function does not appear, it does appear in the evaluation of the sum of the type $\sum_{m>2} \frac{z^{m}}{m+\lambda} \zeta(m, \alpha), \lambda=0,1,2, \ldots$, and this evaluation is essential in all the relevant problems ([18], [45]), including the first proof of Theorem 3 below.

To establish this opinion, we shall give two proofs of [10], Theorem 4.3, which seems the highest summit of the paper (because Theorem 4.1 follows from it by differentiation), and coincides with our Corollary 3 (i) [18]; the first proof depends on the penultimate formula ([18], (9)) of Ramanujan, which we state as Lemma 1 while the second depends on a more antecedent one, i.e. the intermediate formula toward the proof of Proposition $1[16$, p. 10] (reproved as $[10,(2.3)]$, whence follows $[10$, Theorem 4.3$]$ by differentiation), which we state as Lemma 2.

Theorem $3([18]$, Corollary $3(\mathbf{i})=[10]$, Theorem 4.3). For $\lambda \in \mathbb{N} \cup\{0\}$, we have

$$
\begin{aligned}
& \int_{0}^{z} t^{\lambda} \psi(t+a) \mathrm{d} t \\
& =\sum_{k=0}^{\lambda}\left(\begin{array}{l}
\lambda \\
k
\end{array}\right)(-1)^{k} z^{\lambda-k}\left\{\zeta^{\prime}(-k, a+z)-H_{k} \frac{B_{k+1}(a+z)}{k+1}\right\} \\
& -(-1)^{\lambda}\left\{\zeta^{\prime}(-\lambda, a)-H_{\lambda} \frac{B_{\lambda+1}(a)}{k+1}\right\} .
\end{aligned}
$$

(3.1) should be compared with our previous result ([18, Corollary 3]): (i) 
For $\lambda \in \mathbb{N} \cup\{0\}$ and $|z|<\alpha$,

$$
\begin{aligned}
& \int_{0}^{z} t^{\lambda} \psi(\alpha+t) d t \\
& =(-1)^{\lambda} \sum_{r=0}^{\lambda} C_{\lambda}(r, \alpha) \log \Gamma_{r+1}(\alpha+z) / \Gamma_{r+1}(\alpha) \\
& \quad+(-1)^{\lambda} \sum_{l=1}^{\lambda}(-1)^{l}\left\{\left(\begin{array}{l}
\lambda \\
l
\end{array}\right) \zeta^{\prime}(l-\lambda)+\frac{B_{\lambda-l+1}(\alpha)}{l(\lambda-l+1)}\right\} z^{l}+\frac{z^{\lambda+1}}{\lambda+1} H_{\lambda} .
\end{aligned}
$$

(ii) For $\lambda \in \mathbb{N}$

$$
\begin{aligned}
& \lambda \int_{0}^{z} t^{\lambda-1} \log \Gamma(\alpha+t) d t \\
& =z^{\lambda} \log \Gamma(\alpha+z)-(-1)^{\lambda} \sum_{r=0}^{\lambda} C_{\lambda}(r, \alpha) \log \Gamma_{r+1}(\alpha+z) / \Gamma_{r+1}(\alpha) \\
& \quad-(-1)^{\lambda} \sum_{l=1}^{\lambda}(-1)^{l}\left\{\left(\begin{array}{l}
\lambda \\
l
\end{array}\right) \zeta^{\prime}(l-\lambda)+\frac{B_{\lambda-l+1}(\alpha)}{l(\lambda-l+1)}\right\} z^{l}-\frac{z^{\lambda+1}}{\lambda+1} H_{\lambda} .
\end{aligned}
$$

Lemma 1 ([18], (9)). We have

$$
\begin{aligned}
\sum_{m=2}^{\infty} \frac{\zeta(m, \alpha)}{m+\lambda} z^{m+\lambda}= & \sum_{k=0}^{\lambda}\left(\begin{array}{l}
\lambda \\
k
\end{array}\right)\left\{\zeta^{\prime}(-k, \alpha-z)\right. \\
& \left.+H_{k} \zeta(-k, \alpha-z)\right\} z^{\lambda-k} \\
& -\left(\zeta^{\prime}(-\lambda, \alpha)+H_{\lambda} \zeta(-\lambda, \alpha)\right)+\frac{\psi(\alpha)}{\lambda+1} z^{\lambda+1} .
\end{aligned}
$$

First proof of Theorem 3. We start from the Taylor expansion $(|z|<\alpha)$

$$
\begin{aligned}
\psi(z+\alpha) & =\sum_{n=0}^{\infty} \frac{\psi^{(n)}(\alpha)}{n !} z^{n} \\
& =\psi(\alpha)+\sum_{m=2}^{\infty}(-1)^{m} \zeta(m, \alpha) z^{m-1} .
\end{aligned}
$$

Multiplying both sides of (3.3) by $z^{\lambda}$ and integrating over $[0, z]$ with respect to $z$, we deduce that

$$
\begin{aligned}
& \int_{0}^{z} u^{\lambda} \psi(\alpha+u) \mathrm{d} u \\
& =\int_{0}^{z} u^{\lambda} \psi(\alpha) \mathrm{d} u+\sum_{m=2}^{\infty}(-1)^{m} \zeta(m, \alpha) \int_{0}^{z} u^{\lambda+m-1} \mathrm{~d} u \\
& =(-1)^{\lambda} \sum_{m=2}^{\infty} \frac{\zeta(m, \alpha)}{m+\lambda}(-z)^{m+\lambda}+\frac{z^{\lambda+1}}{\lambda+1} \psi(\alpha) .
\end{aligned}
$$


Substituting (3.2) with $-z$ in place of $z$ into (3.4), we obtain

$$
\begin{aligned}
& \int_{0}^{z} u^{\lambda} \psi(\alpha+u) \mathrm{d} u \\
& =(-1)^{\lambda} \sum_{k=0}^{\lambda}\left(\begin{array}{l}
\lambda \\
k
\end{array}\right)\left\{\zeta^{\prime}(-k, \alpha+z)+H_{k} \zeta(-k, \alpha+z)\right\}(-z)^{\lambda-k} \\
& \quad+\frac{\psi(\alpha)}{\lambda+1}(-1)^{\lambda}(-z)^{\lambda+1}+\frac{\psi(\alpha)}{\lambda+1} z^{\lambda+1} \\
& \quad-(-1)^{\lambda}\left(\zeta^{\prime}(-\lambda, \alpha)+H_{\lambda} \zeta(-\lambda, \alpha)\right),
\end{aligned}
$$

which is (3.1).

Lemma 2. ([16,p.10];[10,(2.3)]) For $0 \leq \lambda \in \mathbb{Z}$ we have

$$
\begin{aligned}
(-1)^{\lambda+1} \int_{0}^{z} u^{\lambda} \zeta(s, a+u) \mathrm{d} u \\
=\frac{1}{s-1} \sum_{k=0}^{\lambda}\left(\begin{array}{l}
\lambda \\
k
\end{array}\right) \frac{\Gamma(2-s) k !}{\Gamma(k+2-s)} \zeta(s-k-1, a+z)(-z)^{\lambda-k} \\
\quad-\frac{1}{s-1} \frac{\lambda ! \Gamma(2-s)}{\Gamma(\lambda-2-s)} \zeta(s-\lambda-1, a) .
\end{aligned}
$$

Second proof of Theorem 3. Subtracting $\frac{(-1)^{\lambda+1}}{s-1} \int_{0}^{z} u^{\lambda} \mathrm{d} u$ from the left-side, and $\frac{(-1)^{\lambda+1}}{s-1} \frac{z^{\lambda+1}}{\lambda+1}$ from the right-side, of (3.6), we deduce that

$$
(-1)^{\lambda+1} \int_{0}^{z} u^{\lambda}\left(\zeta(s, a+u)-\frac{1}{s-1}\right) \mathrm{d} u=\frac{1}{s-1} F(s)
$$

where

$$
\begin{aligned}
F(s)= & \sum_{k=0}^{\lambda}\left(\begin{array}{l}
\lambda \\
k
\end{array}\right) \frac{\Gamma(2-s) k !}{\Gamma(k+2-s)} \zeta(s-k-1, a+z)(-z)^{\lambda-k} \\
& -\lambda ! \frac{\Gamma(2-s)}{\Gamma(\lambda+2-s)} \zeta(s-\lambda-1, a)-\frac{(-z)^{\lambda+1}}{\lambda+1} .
\end{aligned}
$$

We are to take the limit of (3.7) as $s \rightarrow 1$. For this we first contend that $F(1)=0$. Indeed,

$$
F(1)=-\sum_{k=0}^{\lambda}\left(\begin{array}{l}
\lambda \\
k
\end{array}\right) \frac{B_{k+1}(a+z)}{k+1}(-z)^{\lambda-k}+\frac{B_{\lambda+1}(a)}{\lambda+1}-\frac{(-z)^{\lambda+1}}{\lambda+1} .
$$

Rewriting $\left(\begin{array}{l}\lambda \\ k\end{array}\right) \frac{1}{k+1}$ as $\frac{1}{\lambda+1}\left(\begin{array}{c}\lambda+1 \\ k+1\end{array}\right)$ and writing $k$ for $k+1$, we derive from (3.9) that

$$
F(1)=-\frac{1}{\lambda+1} \sum_{k=0}^{\lambda+1}\left(\begin{array}{c}
\lambda+1 \\
k
\end{array}\right) B_{k}(a+z)(-z)^{\lambda+1-k}+\frac{B_{\lambda+1}(a)}{\lambda+1},
$$


where we incorporated the last term in (3.4) in the first sum of (3.10). Noting that the fist sum of (3.10) is nothing but the expansion of the Bernoulli polynomial $B_{\lambda+1}(a+z-z)=B_{\lambda+1}(a)$, we conclude $F(1)=0$.

Hence, we may take the limit as $s \rightarrow 1$ of (3.2). On the left side we have $(-1)^{\lambda} \int_{0}^{z} u^{\lambda} \psi(a+u) \mathrm{d} u$ by the Laurent expansion of $\zeta(s, a+u)$, and on the rightside we just differentiate $F(s)$ with respect to $s$, thereby noting the formula

$$
\begin{aligned}
& \left.\left(\frac{\Gamma(2-s)}{\Gamma(k+1-s)}\right)^{\prime}\right|_{s=1} \\
& =\left.\frac{\Gamma(2-s)}{\Gamma(k+2-s)}(-\psi(2-s)+\psi(k+2-s))\right|_{s=1} \\
& =\frac{1}{k !} H_{k},
\end{aligned}
$$

to obtain

$$
\begin{aligned}
F^{\prime}(1)= & \sum_{k=0}^{\lambda}\left(\begin{array}{l}
\lambda \\
k
\end{array}\right)\left\{\zeta^{\prime}(-k, a+z)+H_{k} \zeta(-k, a+z)\right\}(-z)^{\lambda-k} \\
& -\left\{\zeta^{\prime}(-\lambda, a)+H_{\lambda} \zeta(-\lambda, a)\right\}
\end{aligned}
$$

which is equal to $(-1)^{\lambda} \int_{0}^{z} u^{\lambda} \psi(a+u) \mathrm{d} u$. By multiplying by $(-1)^{\lambda}$ completes the proof.

Remark 3.1. In the notation of [10, (3.1), (3.28)],

$$
\begin{aligned}
\zeta^{\prime}(-k, q)+H_{k} \zeta(-k, q) & =\frac{1}{k+1}\left((k+1) \zeta^{\prime}(-k, q)-H_{k} B_{k+1}(q)\right) \\
& =(k+1) ! \psi^{(-k-1)}(q),
\end{aligned}
$$

and our Theorem 3 coincides with Theorem 4.3 of Episona and Moll.

Remark 3.2. (i) Episona and Moll [9] developed the Hurwitz transform

$$
\int_{0}^{1} f(u) \zeta(s, u) \mathrm{d} u
$$

and deduced several results for special types of $f(u)$ which can be expanded into Fourier series as consequences of their Theorem 2.2, which in turn is a consequence of the "Fourier series":

$$
\zeta(s, u)=2 \Gamma(1-s) \sum_{n=1}^{\infty}(2 \pi n)^{s-1} \sin \left(2 \pi n u+\frac{\pi s}{2}\right),
$$

or, more commonly known as the Hurwitz formula. Although they use the name "Fourier series," they do not quote Mikolás' most relevant paper [38], in which 
he gave the simplest proof of (3.13) as the Fourier series, whereby he computed the Fourier coefficients

$$
\int_{0}^{1} \zeta(s, u) e^{-2 \pi i \nu u} \mathrm{~d} u=\frac{\Gamma(1-s)}{(2 \pi i \nu)^{1-s}},
$$

$0<s<1,0 \neq \nu \notin \mathbb{Z}$. From (3.14) we immediately deduce

$$
\begin{aligned}
& \int_{0}^{1} \zeta(s, u) \cos 2 \pi \nu u \mathrm{~d} u-i \int_{0}^{1} \zeta(s, u) \sin 2 \pi \nu u \mathrm{~d} u \\
& =\int_{0}^{1} \zeta(s, u) e^{-2 \pi i \nu u} \mathrm{~d} u=\frac{(2 \pi)^{s} \nu^{s-1}}{4 \Gamma(s)}\left(\frac{1}{\cos \frac{\pi}{2} s}-i \frac{1}{\sin \frac{\pi}{2} s}\right),
\end{aligned}
$$

whence follows Formulas (2.2) and (2.3) of Episona and Moll.

(ii) Although Episona and Moll [9] refer to Mikolás' paper [37] and quote the result

$$
\begin{gathered}
\int_{0}^{1} \zeta(1-s,\{a q\}) \zeta(1-s,\{b q\}) \mathrm{d} q=2 \Gamma^{2}(s) \frac{\zeta(2 s)}{(2 \pi)^{2 s}}\left(\frac{(a, b)}{[a, b]}\right)^{s} \\
((a, b)=\text { g.c.d. of } a \text { and } b, \text { and }[a, b]=\text { l.c.m. })
\end{gathered}
$$

for $\operatorname{Re}(1-s)<\frac{1}{2}$, they do not refer to yet another paper of Mikolás' [36], in which he obtained the result:

$$
\begin{aligned}
& \int_{0}^{1} \zeta(s, u) \zeta\left(s^{\prime}, u\right) \mathrm{d} u \\
& =2(2 \pi)^{s+s^{\prime}-2} \Gamma(1-s) \Gamma\left(1-s^{\prime}\right) \cos \frac{\pi}{2}\left(s-s^{\prime}\right) \zeta\left(2-s-s^{\prime}\right)
\end{aligned}
$$

for $\max \{0, \operatorname{Re} s\}+\max \left\{0, \operatorname{Re} s^{\prime}\right\}<1$; the region of validity wider than that of Episona and Moll who have only $s \leq 0, s^{\prime} \leq 0$. The proof of (3.15) in the case $\operatorname{Re} s \leq 0$ and $\operatorname{Re} s^{\prime} \leq 0$ is based on (3.13) and Fourier analysis (the Parseval formula).

This result of Mikolás', combined with our recent developments of the product of zeta-functions [22], may shed some new light on the asymptotic formula for mean square of zeta-functions. In fact, it looks like the region is one of the excluded one in Katsurada [25] and Katsurada and Matsumoto [28].

The proof of (3.15) in the remaining cases are subtler and essentially depends on the Mellin transform $M(s, z)=\int_{0}^{\infty} u^{z-1} \zeta(s, u) \mathrm{d} u$, proved to exist for $\operatorname{Re} s<$ $1, \max \{0, \operatorname{Re} s\}<\operatorname{Re} z<1$.

Formula (3.6) suggests that considering the partial integral $\int_{0}^{x} u^{z-1} \zeta(s, u) \mathrm{d} u$ may yield a counterpart of (3.15).

\section{The functional equation}

In [17] statements were made about the proof of the functional equation, or the Hurwitz formula (3.13), for the Hurwitz zeta-function, using the absolutely 
convergent Fourier series for $\bar{B}_{2}(t)$ rather than the boundedly convergent Fourier series for $\bar{B}_{1}(t)$. Meanwhile the book of Laurinčikas and Garunkštis [30] has appeared which has rich contents about rather wide spectrum of the theory of the Lerch zeta-function $\phi(\xi, a, s)=\sum_{n=0}^{\infty} \frac{e^{2 \pi i n \xi}}{(n+a)^{s}}$, and we can do no better than referring to it regarding various proofs of the functional equation for $\phi$. We shall therefore restrict mostly to those papers which were not quoted in [30].

As mentioned in Remark 3.2, Mikolás [38] made use of the Fourier series to deduce the functional equation for $\zeta(s, a)$ and in the subsequent paper [39], he applied the same method to prove the functional equation for $\phi(\xi, a, s)$.

Berndt [5] used the boundedly convergent Fourier series to deduce (3.13), which he further applied to $\phi(\xi, a, s)$ to deduce the functional equation in [6], where he gave another proof for it, which was reproduced by [30].

Fine [12] applied Riemann's second method, i.e. the theta-transformation formula (for $\theta_{3}$ ), or what amounts to the same thing, the Poisson summation formula, to prove (3.13), while Apostol [1] deduced (3.13) from the functional equation and the distribution property for $\phi(\xi, a, s)$.

Apostol's paper [2] (cf. also [3]) contains the seemingly most natural proof of the functional equation for $\phi(\xi, a, s)$ based on the transformation formula and the difference equational structure of $\phi$.

As has been developed rather fully in [21], the theta-transformation formula or the modular relation a lá Bochner and the functional equation are equivalent. In this respect, Fine and Apostol would lead to Bochner and may be considered as the prototype of manifestation of the zeta-function associated to prehomogeneous vector spaces.

We remark, however, that although in the above mentioned papers, Lipschitz [34], Lerch [32], Hurwitz [14] are referred to, but are neither Malmstén [35] nor Schlömilch [44], who gave the functional equation for some $L$-functions (the $L$ function modulo 4, to be precise), nor the paper of Euler. In this regard we must take into account Weil's paper [46], which gives a translation and comments on Eisenstein's copy of Gauss' Disquisitiones, especially the last page (dated 1849) inserted by the binder. On that page, Eisenstein made an "unmotivated" application of the Poisson summation formula to prove the functional equation for $\phi(\xi, a, s)$ from which he deduces that for $L$-function mod 4 . His argument precedes Oberhettinger [40] by 107 years in that he uses the Fourier transform

$$
\int_{0}^{\infty} e^{2 \pi i x y} x^{q-1} \mathrm{~d} x
$$

while Oberhettinger produces the proof by using the Laplace transform. The Fourier transform also the basis of Mikolás' proof [38]. Here we may enjoy a happy encounter of some of the greatest unsimultaneous mathematicians of all time, Gauss, Eisenstein and Weil. We are also fascinated by Weil's imagination on the source of Riemann's paper.

We are indebted to Sato's paper [43] for this paper of Weil; without Sato's, we may have missed it, and indeed, in no other places, this discovery of Eisenstein has been presented. E.g. in Grosswald, the Lipschitz transformation formula (i.e. 
the functional equation) is proved by the Poisson summation formula, which is in principle the same as Eisenstein's proof.

Sato's paper (cf. [21] as well) contains a very nice list of functional equations that follow from the theta-transformation formula and some other deep insight.

In $\S 5$ of [17] we posed a problem of proving the functional equation (3.13) by completing the integral $\int_{0}^{\infty} \bar{B}_{1}(t)(t+a)^{u-1} \mathrm{~d} t$ and using some transformation formula for confluent hypergeometric functions. We have not found such a hypergeometric functional proof, but we can present a more high-brow proof using the Fourier series for the Dirac delta function $\delta(s)$ by completing the incomplete gamma functions (in a sense completing the above integral, thus our guess half hit the points).

Our starting point is thus the combination of (41) and (43) of [17] (where we write $s$ for $-u$ )

$$
\begin{aligned}
\zeta(s, a)= & \frac{1}{a^{s-1}} \sum_{n=1}^{\infty}\left(\frac{e^{-2 \pi i n a}}{(-2 \pi i n a)^{1-s}} \Gamma(1-s,-2 \pi i n a)\right. \\
& \left.+\frac{e^{2 \pi i n a}}{(2 \pi i n a)^{1-s}} \Gamma(1-s, 2 \pi i n a)\right) \\
+ & \frac{1}{2 a^{s}}+\frac{1}{a^{s-1}} \frac{1}{s-1} .
\end{aligned}
$$

We use the incomplete gamma function $\gamma(s, a)$ of the first kind

$$
\gamma(s, a)=\int_{0}^{a} e^{-u} u^{s-1} \mathrm{~d} u=a^{s} \int_{0}^{1} e^{-a u} u^{s-1} \mathrm{~d} u
$$

and complete $\Gamma(1-s, a)$ to write $\Gamma(1-s, a)=\Gamma(1-s)-\gamma(1-s, a)$. Thus

$$
\begin{gathered}
\zeta(s, a)=\Gamma(1-s) \sum_{n=1}^{\infty}\left(\frac{e^{-2 \pi i n a}}{(-2 \pi i n)^{1-s}}+\frac{e^{2 \pi i n a}}{(2 \pi i n)^{1-s}}\right)+\frac{1}{2 a^{s}}+\frac{1}{a^{s-1}} \frac{1}{s-1} \\
-\frac{1}{a^{s-1}} \sum_{n=1}^{\infty}\left(e^{-2 \pi i n a} \int_{0}^{1} e^{2 \pi i n a u} u^{-s} \mathrm{~d} u\right. \\
\left.\quad+e^{2 \pi i n a} \int_{0}^{1} e^{-2 \pi i n a u} u^{-s} \mathrm{~d} u\right) .
\end{gathered}
$$

We invert the order of summation and integration in the last term and consider the series $\sum_{n=-\infty}^{\prime \infty} e^{-2 \pi i n a(u-1)}$ as the Fourier series for $\delta(a(u-1))-1$. Then we are left with the integration $(\sigma<0)$

$$
-\int_{0}^{1} \delta(a(u-1)) u^{-s} \mathrm{~d} u+\int_{0}^{1} u^{-s} \mathrm{~d} u=-\frac{1}{2 a}-\frac{1}{s-1} .
$$

Hence the last term is $-\frac{1}{2 a^{s}}-\frac{1}{s-1} \frac{1}{a^{s-1}}$, which cancels the second term and we finally arrive at

$$
\zeta(s, a)=\frac{\Gamma(1-s)}{(2 \pi)^{1-s}}\left(e^{\frac{1-s}{2} \pi i} \ell_{1-s}(1-a)+e^{-\frac{1-s}{2} \pi i} \ell_{1-s}(a)\right),
$$


which is equivalent to (3.13).

\section{References}

[1] T. M. Apostol, "Remark on the Hurwitz zeta function," Proc. Amer. Math. Soc. 2 (1951), 690-693.

[2] T. M. Apostol, "On the Lerch zeta-function," Pacific J. Math. 1 (1951), 161-167.

[3] T. M. Apostol, "Addendum to 'On the Lerch zeta-function'," Pacific J. Math. 2 (1952), 10.

[4] E. W. Barnes, "On the theory of the multiple gamma functions," Trans. Cambridge Philos. Soc. 19 (1904), 374-425.

[5] B. C. Berndt, "On the Hurwitz zeta-function," Rocky Mount. J. Math. 2 (1972), 151-157.

[6] B. C. Berndt, "Two new proofs of Lerch's functional equation," Proc. Amer. Math. Soc. 32 (1972), 403-408.

[7] B. C. Berndt, "The gamma function and the Hurwitz zeta-function," Amer. Math. Monthly 92 (1985), 126-130.

[8] C. Deninger, "On the analogue of the formula of Chowla and Selberg for real quadratic fields," J. Reine Angew. Math. 351 (1984), 171-191.

[9] O. Episona and V. H. Moll, "On some integrals involving the Hurwitz zetafunction: Part 1," The Ramanujan J. 6 (2002), 159-188.

[10] O. Episona and V. H. Moll, "On some integrals involving the Hurwitz zetafunction: Part 2," The Ramanujan J. 6 (2002), 449-468.

[11] A. Erdélyi, W. Magnus, F. Oberhettinger and F. G. Tricomi (The Bateman Manuscript Project), Higher Transcendental Functions, Vol. I, McGrawHill, New York, Toronto, and London, 1953.

[12] N. J. Fine, "Note on the Hurwitz zeta-function," Proc. Amer. Math. Soc. 2 (1951), 361-364.

[13] E. Grosswald, Representations of integers as sums of two squares, Springer Verlag, New York-Berlin-Heidelberg-Tokyo 1985.

[14] A. Hurwitz, "Einige Eigenschaften der Dirichlet'schen Funktionen $F(s)=$ $\sum\left(\frac{D}{n}\right) \cdot \frac{1}{n^{s}}$, die bei der Bestimmung der Classenanzahlen binärer quadratischer Formen auftreten," Zeitschrift f. Mathematik u. Physik 27 (1882), 86101.

[15] S. Kanemitsu, "Some sums involving Farey fractions," RIMS Kôkyûroku 958 (1996), 14-22. 
[16] S. Kanemitsu, M. Katsurada and M. Yoshimoto, "On the Hurwitz-Lerch zeta-function," Aequationes Math. 59 (2000), 1-19.

[17] S. Kanemitsu, H. Kumagai, H. M. Srivastava and M. Yoshimoto, "Some integral and asymptotic formulas associated with the Hurwitz zeta-function," Appl. Math. Comput. 154 (2004), 641-664.

[18] S. Kanemitsu, H. Kumagai and M. Yoshimoto, "Sums involving the Hurwitz zeta function," Ramanujan J. 5 (2001), 5-19.

[19] S. Kanemitsu, H. Kumagai and M. Yoshimoto, "On rapidly convergent series expressions for zeta- and $L$-values, and log sine integrals," Ramanujan J. 5 (2001), 91-104.

[20] S. Kanemitsu, Y. Tanigawa and M. Yoshimoto, "On rapidly convergent series for the Riemann zeta-values via the modular relation," Abh. Math. Sem. Univ. Hamburg 72 (2002), 187-206.

[21] S. Kanemitsu, Y. Tanigawa and M. Yoshimoto, "Ramanujan's formula and modular forms," in Number-theoretic methods - future trends (ed. by Shigeru Kanemitsu and Chaohua Jia), Kluwer Academic Publ., 2002, pp. 159-212.

[22] S. Kanemitsu, Y. Tanigawa and M. Yoshimoto, "Structural elucidation of the mean square of the Hurwitz zeta-function," J. Number Theory 120 (2006), 101-119.

[23] S. Kanemitsu, Y. Tanigawa and M. Yoshimoto, "Determination of some lattice sum limits," J. Math. Anal. Appl. 294 (2004), 7-16.

[24] S. Kanemitsu and H. Tsukada, Vistas of special functions, to appear.

[25] M. Katsurada, "An application of Mellin-Barnes' type integrals to mean square of Lerch zeta-functions," Collect. Math. 48 (1997), 137-153.

[26] M. Katsurada, "Power series and asymptotic series associated with the Lerch zeta-function," Proc. Japan Acad. Ser. A Math. Sci. 74 (1998), 167170 .

[27] M. Katsurada, "An application of Mellin-Barnes type integrals to the mean square of L-functions," Liet. Mat. Rink. 38 (1998), 98-112.

[28] M. Katsurada and K. Matsumoto, "Explicit formulas and asymptotic expansions for certain mean square of Hurwitz zeta functions I," Math. Scand. 78 (1996), 161-177.

[29] D. Klusch, "On the Taylor expansion of the Lerch zeta-function," J. Math. Anal. Appl. 170 (1992), 513-523.

[30] A. Laurinčikas and R. Garunkštis, The Lerch zeta-function, Kluwer Academic Publ., Dordrecht-Boston-London 2002. 
[31] A. F. Lavrik, "An aspproximate functional equation for the Dirichlet $L$ function," Trady Moskov. Math. Obšč 18 (1968), 91-104 = Trans. Moskow Math. Soc. 18 (1968), 105-115.

[32] M. Lerch, "Note sur la fonction $\mathfrak{K}(w, x, s)=\sum_{k=0}^{\infty} \frac{e^{2 k \pi i x}}{(w+k)^{s}}$," Acta Math. 11 (1887), 19-24.

[33] H.-L. Li and M. Toda, "Elaboration of some results of Srivastava and Choi," J. Anal. Appl. 25 (2006), 517-533.

[34] R. Lipschitz, "Untersuchungen der Eigenschaften einter Gattung von unendlichen Reihen," J. Reine Angew. Math. 105 (1889), 127-156.

[35] C. J. Malmstén, "De integralibus quibusdam definitis, seriebusque infinitis," J. Reine Angew. Math. 38 (1849), 1-39.

[36] M. Mikolás, "Mellinsche Transformation und Orthogonalität bei $\zeta(s, u)$; Verallgemeinerung der Riemannschen Funktionalgleichung von $\zeta(s)$, , Acta Sci. Math. (Szeged) 17 (1956), 143-164.

[37] M. Mikolás, "Integral formulae of arithmetical characteristics relating to the zeta-function of Hurwitz," Publ. Math. (Debrecen) 5 (1957-58), 44-53.

[38] M. Mikolás, "A simple proof of the functional equation for the Riemann zeta-function and a formula of Hurwitz," Acta Sci. Math. (Szeged) 18 (1957), 261-263.

[39] M. Mikolás, "New proof and extension of the functional equation for Lerch's zeta-function," Ann. Univ. Sci. Budapest 14 (1971), 111-116.

[40] F. Oberhettinger, "Note on the Lerch zeta function", Pacific J. Math. 6 (1956), 117-120.

[41] Cheng-Dong Pan and Cheng-Biao Pan, Elements of Analytic Number Theory, Science Press, Beijing, 1991.

[42] H. Rademacher, Topics in Analytic Number Theory, Springer-Verlag, Berlin, 1973.

[43] F. Sato, "Searching for the origin of the theory of PHV (Prehomogeneous Vector Spaces)," Annual Meeting of the Math. Soc. Japan 1992.

[44] O. Schlömilch, "Uebungsaufgaben für Schuler, Lehrsatz von dem Herrn Prof. Dr. SCHLÖMILCH," Arch. Math. u. Phys. (Grunert's Archiv), 12 (1849), p. 415.

[45] H. M. Srivastava and J. Choi, Series Associated with the Zeta and Related Functions, Kluwer Academic Publishers, Dordrecht, Boston, and London, 2001. 
[46] A. Weil, "On Eisenstein's copy of the Disquisitiones," Advanced Studies in Pure Mathematics 17, 1989 Algebraic Number Theory - in honor of K. Iwasawa, pp. 463-469.

[47] J. R. Wilton, "A proof of Burnside's formula for $\log \Gamma(x+1)$ and certain allied properties of Riemann's $\zeta$-function," Messenger Math. 52 (1922/1923), 90-93.

[48] J. R. Wilton, "A note on the coefficients in the expansion of $\zeta(s, x)$ in powers of $s-1$," Quart. J. Pure Appl. Math. 50 (1927), 329-332.

[49] X.-Y. Wang, S. Kanemitsu and Y. Tanigewa, "An application of the Euler digamma function," to appear. 
Shigeru Kanemitsu

Graduate School of Advanced Technology, University of Kinki, lizuka, Fukuoka, 820-8555, Japan

e-mail: kanemitu@fuk.kindai.ac.jp

Yoshio Tanigawa

Graduate School of Mathematics, Nagoya University,

Nagoya, 464-8602, Japan

e-mail: tanigawa@math.nagoya-u.ac.jp

Haruo Tsukada

Graduate School of Advanced Technology, University of Kinki, lizuka, Fukuoka, 820-8555, Japan

e-mail: tsukada@fuk.kindai.ac.jp

Masami Yoshimoto

Graduate School of Mathematics, Nagoya University,

Nagoya, 464-8602, Japan

e-mail: x02001n@math.nagoya-u.ac.jp

Received on 17-10-06

Accepted on 18-07-07 